\title{
Resistant Starch Regulates Gut Microbiota: Structure, Biochemistry and Cell Signalling
}

\author{
Xiaoping Yang ${ }^{a, b}$ Kwame Oteng Darko ${ }^{a}$ Yanjun Huang ${ }^{a, b}$ Caimei He $\mathrm{H}^{\mathrm{a}, \mathrm{b}}$ \\ Huansheng Yang ${ }^{b}$ Shanping He ${ }^{b}$ Jianzhong Li $^{b}$ Jian Lic Berthold Hocherc,d \\ Yulong Yin ${ }^{\mathrm{b}, \mathrm{e}}$
}

\begin{abstract}
aDepartment of Pharmacy, School of Medicine, Hunan Normal University, Changsha, China; ${ }^{b}$ Animal Nutrition and Human Health Laboratory, College of Life Sciences, Normal University, Changsha, China; 'Department of Basic Medicine, School of Medicine, Hunan Normal University, Changsha, China;

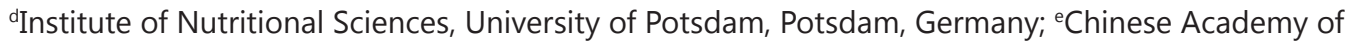
Science, Institute of Subtropical Agriculture, Research Center for Healthy Breeding of Livestock and Poultry, Hunan Engineering and Research Center of Animal and Poultry Science and Key Laboratory for Agroecological Processes in Subtropical Region, Scientific Observation and Experimental Station of Animal Nutrition and Feed Science in South-Central, Ministry of Agriculture, Changsha, China
\end{abstract}

\section{Key Words}

Resistant starch • Gut microbiota • Nutrition

\begin{abstract}
Starch is one of the most popular nutritional sources for both human and animals. Due to the variation of its nutritional traits and biochemical specificities, starch has been classified into rapidly digestible, slowly digestible and resistant starch. Resistant starch has its own unique chemical structure, and various forms of resistant starch are commercially available. It has been found being a multiple-functional regulator for treating metabolic dysfunction. Different functions of resistant starch such as modulation of the gut microbiota, gut peptides, circulating growth factors, circulating inflammatory mediators have been characterized by animal studies and clinical trials. In this mini-review, recent remarkable progress in resistant starch on gut microbiota, particularly the effect of structure, biochemistry and cell signaling on nutrition has been summarized, with highlights on its regulatory effect on gut microbiota.

\section{Introduction}

Starch is a major energy source to all living cells with a mixture of $\alpha$-glucan, polysaccharide, amylose and amylopectin in a proportion of $80-90 \%$ of all polysaccharides. The nutritional property of starch is related to its rate and extent of digestion and absorption in the small intestine. To label its nutritional features, starch is classified as rapidly digestible, slowly digestible and resistant starch [1]. Rapidly digestible starch (RDS) is the starch fraction that causes a sudden increase in the blood glucose level after ingestion, and slowly digestible starch (SDS) is a starch fraction that is digested completely in the small intestine at a slower rate as compared with rapidly digestible starch. Resistant starch (RS) is the starch portion

Xiaoping Yang and Yulong Yin, Ph. D. Department of Pharmacy, School of Medicine, Hunan Normal University, Changsha, Hunan, 410013 (China), and Institute of Subtropical Agriculture, Chinese Academy of Sciences, Changsha, Hunan, 410125 (China) 
that is not absorbed in the small intestine of healthy individuals but is fermented in the large intestine. One of the most important chemical features of these different starch molecules is that high amylose content and specific structures of amylopectin molecule are necessary for the production of resistant starch of hydrothermally treated starch. [2]. A number of factors contribute to the ability for starch to be able to escape digestion in the small intestine. Based on these factors resistant starch has been categorized into four. Resistant Starch 1(RS1): physically inaccessible to digestion by entrapment in a nondigestible matrix; Resistant Starch 2 (RS2): ungelatinized starch; Resistant Starch 3 (RS3); retrograded starch and Resistant Starch 4 (RS4): chemically modified starch [3].

Previously, Yin et al. highlighted the nutritional and health functions of carbohydrates for pigs [4]. However, several remarkably progress in non-digestible starch in nutrition and food research area have been revealed since then. Particularly, benefits from the recently remarkable progress of microbiota, the nutritional effects of carbohydrates on the gut have been notably recognized. Thus, we summarize recent breakthroughs, highlighting the biochemistry and cell signaling of RS.

\section{Chemical Characteristics of Various RS}

RS has its chemical structural features such that amylopectin and amylose are the two major components determining its biochemical and nutritional properties [5]. Chemical structural specificities of starch polysaccharides (amylose and amylopectin) can be evaluated by methylation analysis, electrospray mass spectrometry (ESI-MS), tandem mass spectrometry (ESI-MS/MS) and anionic chromatography. Morphological determination via microscopy could be used for determining the loss of birefringence of starch as a marker of observable granule disruption during processing.

$100 \%$ amylopectin corn starch is essentially $100 \%$ digestible. Hi-maize product is considered a high-amylose maize starch. RDS can be digested to glucose within 20 min after initiation of treatment of the high-amylose maize starch with amylase and other enzymes in an in vitro assay. SDS is digested to glucose within 20-120 min. RS is the starch that is digested after 120 min [5].

Protein in rice interacts with starch, binding to amylose or amylopectin, which causes a positive correlation between protein and RS content[6]. Lipid in rice affects the RS content by complexing with amylose [7]. Amylose content is negatively correlated with RDS and positively correlated with SDS and RS. Similar relationships between amylose content and starch digestibility in rice have been reported earlier where Rashmi and Urooj showed that the amylose content was inversely related to the amount of RDS and Panlasigui et al. also suggested that the amylose content influenced in vitro starch digestion as well as glycemic response in humans. Thus, in general, amylose in rice starch granules is resistant to digestion by enzyme $[8,9]$.

When the linear starch chain is in a helical-complex structure with complexed fatty acid in the cavity of the helix, a starch binding and cleavage by amylase are prevented. In addition, the amylase-lipid complex also entangles amylopectin molecules, restricting the swelling of starch granules and enzyme hydrolysis [10].

\section{Health Benefits of Resistant Starch}

Evidence from both animal models and humans agrees with the role o f resistant starches in improving a variety of metabolic features [11]. Using pig as an animal model, Heo et al. found that feeding a diet containing resistant potato starch showed benefits of gastrointestinal tract traits and growth performance of weaned pigs [12]. Resistant potato starch supplementation decreased ileal and cecal digesta $\mathrm{pH}$. Supplementing a weaned pig diet with more than $0.5 \%$ resistant potato starch independent of the mode of delivery has 


\section{Cellular Physiology Cell Physiol Biochem 2017;42:306-318 and Biochemistry Published online: May 22, $2017 \quad \begin{aligned} & \text { DOI: 10.1159/000477386 } 2017 \text { The Author(s). Published by S. Karger AG, Basel } \\ & \text { www.karger.com/cpb }\end{aligned}$ \\ Yang et al.: Resistant Starch Regulates Gut Microbiota to Control Nutritional Events}

the potency to enhance outcome characteristic of a functional gut without adverse effects on growth.

Karimi et al. initiated a randomized, controlled, parallel-group clinical trial to examine the beneficial effect of resistant starch on type 2 diabetes, one of the most important metabolic diseases. With a total of 56 female subjects, this clinical trial demonstrated that supplementation with resistant starch type II may improve glycemic status, endotoxemia and markers of oxidative stress in patients with type 2 diabetes mellitus [13].

Sun et al. investigated metabolomic and transcriptomic responses in the livers of pigs to evaluate the effects of resistant starch on the body's metabolism at the extra intestinal level [14]. The results showed the raw potato starch diet decreased cholesterol and palmitic acid as well as increased 3-hydroxybutyric acid. In addition, 2-ketoglutaric acid and glucose6-phosphate were increased although pyruvic acid was decreased in the raw potato starch group. This resistant starch diet decreased fatty acid intake and synthesis and increased fatty acid oxidation and glycerol-phospholipid synthesis [14].

Bean, another form of resistant starch, led to decrease in energy intake, waist circumference, systolic blood pressure and HbA1c and to improvements in blood glucose control and insulin sensitivity [15]. Commonly, inulin is used as a prebiotic, fat replacer, sugar replacer, texture modifier and for the development of functional foods in order to improve health due to its beneficial role in gastric health [16].

It is not new that the consumption of resistant starch is beneficial to cardiovascular diseases. Particularly, it has been shown that resistant starch is associated with cardiovascular risk reduction through multiple mechanisms [17]. $\beta$-glucan from oats or barley, or a combination of whole oats and barley, and soluble fiber from psyllium reduces the risk of coronary heart disease. Inulin-type fructans added to foods and beverages may modestly decrease serum triacylglycerols.

Using colonic experimental colitis animal model induced by dextran sulfate sodium, the effects of consumption of a diet high in red meat with adding the resistant starch were examined and the addition of resistant starch exhibited a great benefit on alleviating dextran sulfate sodium plus red meat induced inflammation, indicating that improvement of colonic colitis by resistant starch attributes to its function of anti-inflammation [18].

Dietary treatment on patients who have impaired fasting glucose, impaired glucose tolerance or newly diagnosed type 2 diabetes with resistant starch reduced fasting insulin and insulin resistance, postprandial glucose and insulin levels [19]. A study in a singleblinded, randomized, parallel nutritional intervention for 20 insulin resistant subjects has demonstrated therapeutic effect of resistant starch treatment by showing improved insulin sensitivity, waist circumference and fat storage in tibialis muscle [20]. Furthermore, high resistant starch diet is able to slow down CKD progression and attenuate oxidative stress and inflammation [21]. The digestive system for various starch and potential functions of improvement of health are highlighted in Fig. 1.

\section{Biochemical Functions of Resistant Starch}

In a study conducted by Karimi et al., RS2 caused a significant decrease in the levels of MDA, glycosylated hemoglobin, insulin, homeostasis model of insulin resistance and endotoxin, but a significant increase in TAC and glutathione peroxidase. No significant changes were observed in fasting plasma glucose, quantitative insulin sensitivity check index, hs-CRP, superoxide dismutase, catalase and uric acid in the RS2 group as compared with the control group [13].

RS4 is able to reduce cholesterols, fasting glucose, glycosylated haemoglobin, and proinflammatory markers in the blood as well as waist circumference and percentage of body fat [22]. Sun et al. reported that raw potato starch dramatically affected starch and sucrose metabolites, amino acid turnover or protein biosynthesis, lipid metabolites, glycolysis, the pentose phosphate pathway, inositol phosphate metabolism, and nucleotide metabolism 
Fig. 1. Types of starch and parts of the digestive system where they are absorbed and functions of resistant fortreating metabolic dysfunction.

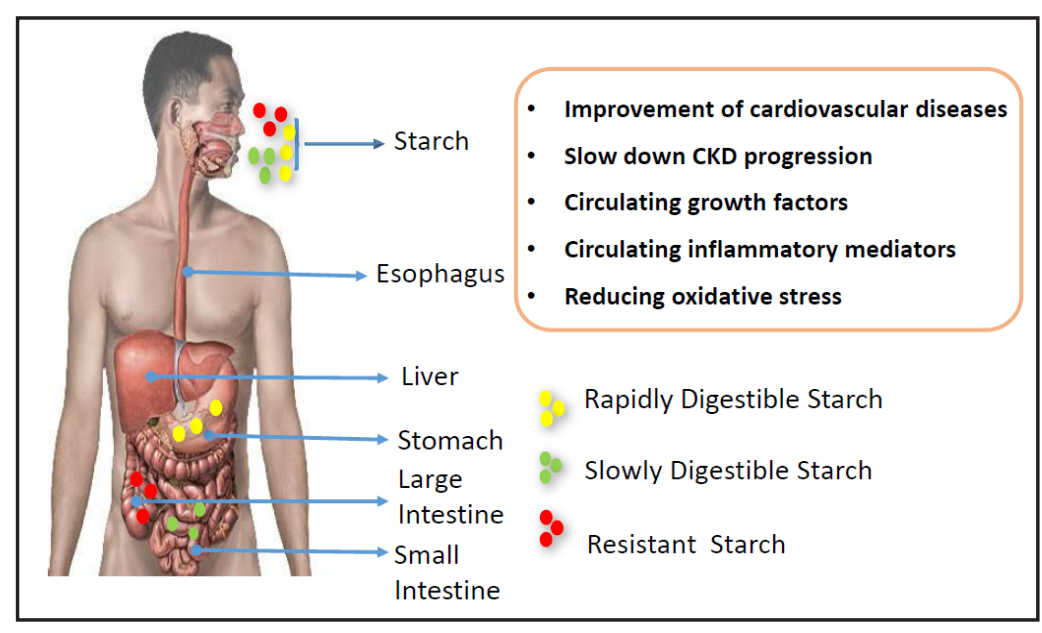

[23]. Furthermore, resistant starch increases the production of glucose-6-phosphate, maltose, arachidonic acid, 9, 12-octadecadienoic acid, oleic acid, phosphate, but decreases the formation of $\alpha$-aminobutyric acid [23].

Deng et al. revealed that treatment of high level of resistant starch diet decreased plasma contents of glucose, insulin, lactic acid. Furthermore, the fractional synthesis rate in the pancreas, spleen, duodenum, jejunum, ileum and colon in a corn group were much higher in high level resistant starch treated group compared to low level resistant starch diet fed group [24].

Tang et al. found that dietary supplementation of chitosan exhibited the enhancement effects on serum growth hormone and insulin-like growth factor-I levels, hepatic and long gissimus muscle IGF-I mRNA expression and increased hepatic and muscle IGF-1 mRNA levels in early-weaned piglets [25].

Notably, it's been disclosed that dietary chitosan oligosaccharide profoundly alleviates lipopolysaccharide-induced inflammatory injuries in the jejunum and ileum. This beneficial effect of chitosan oligosaccharide is related to the increase of calcium-sensing receptorand PLC $\beta 2$ protein expressions and decrease of $\mathrm{p}-\mathrm{NF}-\kappa \mathrm{B}$ p65, IKK $\alpha / \beta$, and IкB protein levels [26].

Another finding by Lyte et al., showed that animals fed on resistant starch diet displayed observable weight gain and anxiety-like behavior [27]. This result raised an interesting phenomenon that intake of resistant starch might cause undesirable effects such as increased anxiety-like behavior.

Toden et al. conducted the first measurement of circulating factors including malondialdehyde (MDA), leptin, insulin-like growth factor-I (IGF-I), insulin, matrix metalloproteinase-2 (MMP-2), tissue inhibitor of MMP-2 (TIMP-2), interleukins (IL), and short-chain fatty acids to explore the effect of resistant starch on nutrition [28]. Leptin concentrations were lowered by high amylose maize starch. MMP-2 and TIMP2 concentrations were higher while IL-1beta and IL-12 concentrations were lowered by high amylose maize starch feeding. Colonic DNA strand breakage was positively associated with circulating leptin and MDA concentrations as well as tissue MDA concentrations and negatively associated with plasma TIMP-2 concentration. MMP-2 and TIMP-2 positively correlated with hepatic portal butyrate levels but leptin concentrations correlated negatively. Diets high in resistant starch could influence cancer initiation or progression by changes in circulating levels of hormones and other factors [28]. In a previous mentioned study Kwak et al. consistently convinced that MDA is a promising biomarker to determine the improvement of endothelial functions with diets containing rice with resistant starch [19].

\section{Unique Functions of Resistant Starch on Microbiomes}

"Microbiota" is the term used to describe the community of microorganisms (bacteria, viruses, and fungi) that normally live in or on a given organ in the body [37]. The gut 


\section{Cellular Physiology Cell Physiol Biochem 2017;42:306-318

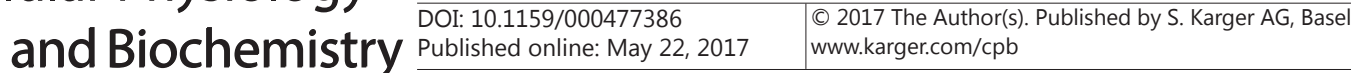 \\ Yang et al.: Resistant Starch Regulates Gut Microbiota to Control Nutritional Events}

microbiota contains roughly one quadrillion cells, at least ten times as many cells as does the body itself. The gastrointestinal tract microbiota contributes to the development and differentiation of the mammalian immunesystem. The composition of the microbiota affects immune responses and susceptibility to infection by intestinal pathogensand development of allergic and inflammatory bowel diseases[29].

Hundreds of bacterial species make up the mammalian intestinal microbiota. Bacteria in the mammalian intestine extremely possess a far larger repertoire of degradative enzymes and metabolic capabilities than their hosts [30]. Certain dominant species, notably among the bacteroidetes, are known to possess very large numbers of genes that encode carbohydrate active enzymes and can switch readily between different energy sources in the gut depending on availability. Nevertheless, more nutritionally specialized bacteria appear to play critical roles in the community by initiating the degradation of complex substrates such as plant cell walls, starch particles and mucin.

Venkataraman et al. have shown a beneficial function of resistant starch on microbiomes which is associated with the increase of butyrate amount in the large intestine [31]. Butyric acid contributes to host health by facilitating maintenance of epithelial integrity, regulating inflammation, and influencing gene expression in colonocytes. Majority of individuals had the increased butyric acid after administration of resistant starch but a small portion had no responses of butyric acid enhancement observed. The authors demonstrated that it was critical that an individual had the special bacteria to degrade the resistant starch for the increase of butyric acid.

Upadhyaya et al. investigated the effect of resistant starch type IV on gut microbiota composition and short-chain fatty acid concentrations with host immune metabolic functions [22]. Higher SCFAs in feces, such as butyrate, propionate, valerate, isovalerate, and hexanoate were observed after resistant starch type IV-intake using gas chromatographymass spectrometry determination.

As mentioned earlier, raw potato starch dramatically induces changes in microbial composition and microbial metabolites [23]. Coprococcus, Ruminococcus, and Turicibacter increased significantly, while Sarcina and Clostridium decreased in relative abundances in the hindgut of pigs fed raw potato starch. A Pearson's correlation analysis confirmed that Ruminococcus and Coprococcus were positively correlated with glucose-6-phosphate, maltose, arachidonic acid, 9, 12-octadecadienoic acid, oleic acid, phosphate, but negatively correlated with $\alpha$-aminobutyric acid. Thus, raw potato starch not only alters the composition of the gut microbial community but also modulates the metabolic pathway of microbial metabolism [23].

Ruminococcus bromii is a dominant member of the human gut microbiota that plays a key role in releasing energy from dietary starches that escape digestion by host enzymes via its exceptional activity against particulate resistant starches [32]. Genomic analysis of R. bromii shows that it is highly specialized, with 15 of its 21 glycoside hydrolases belonging to one family. Six GH13 amylases that carry signal peptides were detected by proteomic analysis in R. bromii cultures [32].

Edwards et al. compared the effects of two porridge meals prepared from wheat endosperm with different degrees of starch bioaccessibility on postprandial metabolism to study physical biochemical disassembly of cereal food during digestion [33]. The postprandial changes in blood glucose, insulin, C-peptide, lipids and gut hormones and on the resistant starch content of ileal effluent were determined to gain insight into the structural and biochemical breakdown of the test meals during gastroileal transit. Undigested food in the ileal output was examined microscopically to identify cell walls and encapsulated starch. Microscopic examination revealed a progressive loss of starch from the periphery toward the particle core. Blood glucose, insulin, C-peptide, and glucose-dependent insulinotropic polypeptide concentrations were significantly lower after consumption of the coarse porridge than after the smooth porridge. In vitro, starch digestion was slower in the coarse porridge than in the smooth porridge. In vivo, the structural integrity of coarse particles of wheat endosperm was retained during gastroileal transit [33]. 


\section{Cellular Physiology Cell Physiol Biochem 2017;42:306-318

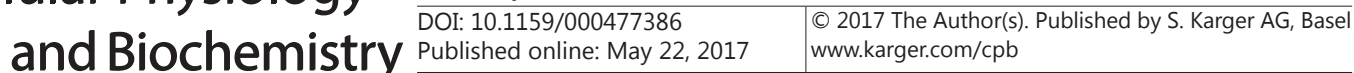 \\ Yang et al.: Resistant Starch Regulates Gut Microbiota to Control Nutritional Events}

Another work reported that changing the gut microbiota using inulin modulates the progression of leukemia and related metabolic disorders [34]. Using leukemia mice model, inulin supplementation increased the portal SCFA propionate and butyrate, and decreased cancer cell invasion in the liver. Inulin treatment increased acetate in the caecal content, changed the fatty acid profile inside adipose tissue and counteracted the induction of markers controlling $\beta$-oxidation, thereby hampering fat mass loss.

When mice were fed with fibers that differed in carbohydrate composition or a control diet, a distinct reaction of the host and microbiota to resistant starch was observed via determination of colonic gene expression profiles and luminal microbiota composition by microarray techniques [35]. Not surprisingly, mice were fed a fiber that was not completely fermented in the colon, whereas the other fibers induced similar responses on gene expression and microbiota. Consistent associations were revealed between fiber-induced enrichment of Clostridium cluster IV and XIVa representatives, and changes in mucosal expression of genes related to energy metabolism.

Ingestion of a whole-grain cereal food product, rich in resistant starch has been shown to affect release of endogenous gut hormones and ameliorate appetite control and glycaemic control, based on randomized cross-over study in healthy middle-aged subjects [36]. The analysis of blood glucose regulation, gastrointestinal hormones, markers of inflammation and markers of colonic fermentation demonstrated that intervention with the representative diet of resistant starch, barley kernel-based bread increased gut hormones p-glucagon-like peptide- 1 and -2 in plasma[36]. Breath $\mathrm{H}_{2}$ excretion and short chain fatty acid concentrations of fasting serum (s) were increased, and glucose and insulin responses were decreased after resistant starch intervention. Insulin sensitivity index was also improved after resistant starch treatment. It is clear that $3 \mathrm{~d}$ of intervention with resistant starch increased systemic levels of gut hormones involved in appetite regulation, metabolic control and maintenance of gut barrier function, as well as improved markers of glucose homoeostasis in middle-aged subjects.

The effects of a diet high in resistant starch on luminal microbiota composition, luminal SCFA concentrations, and the expression of host genes involved in SCFA uptake, SCFA signaling, and satiety regulation in mucosal tissue obtained from small intestine, cecum, and colon were studied using 20 adult female pigs. Resistant starch was completely degraded in the cecum. In the colon these included the stimulation of the healthy gut-associated butyrate-producing Faecal bacterium prausnitzii, whereas potentially pathogenic members of the Gammaproteobacteria, including Escherichia coli and Pseudomonas spp., were reduced in relative abundance. Cecal and colonic short-chain fatty acids concentrations were significantly greater in resistant starch-fed pigs, and cecal gene expression of monocarboxylate transporter 1 (SLC16A1) and glucagon (GCG) was induced by resistant starch. These results demonstrated that resistant starch modulates microbiota composition, short-chain fatty acids concentrations, and host gene expression in pig intestine [37].

Microbial fermentation of complex non-digestible dietary carbohydrates and hostderived glycans in the human intestine has important consequences for health. Certain dominant species, notably among the Bacteroidetes, are known to possess very large numbers of genes that encode carbohydrate active enzymes and can switch readily between different energy sources in the gut depending on availability. Nevertheless, more nutritionally specialized bacteria appear to play critical roles in the community by initiating the degradation of complex substrates such as plant cell walls, starch particles and mucin. Microbial degradation of diverse carbohydrates in the gut has been summarized and the impact of dietary carbohydrateson human health has been discussed via understanding of the complex relationship between diet composition, the gut microbiota and metabolic outputs [38]. The beneficial effect of resistant starch on gut environment is summarized in Fig. 2. 


\section{Cellular Physiology

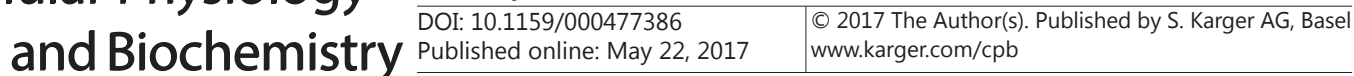 \\ Yang et al.: Resistant Starch Regulates Gut Microbiota to Control Nutritional Events}

Fig. 2. The beneficial effect of resistant starch on gut environment. Dietary resistant starch enhances population of Ruminococcus Bromii in gut microbiota, increases the concentration of short-chain fatty acids, improves microbial metabolism and increases insulin sensitivity.

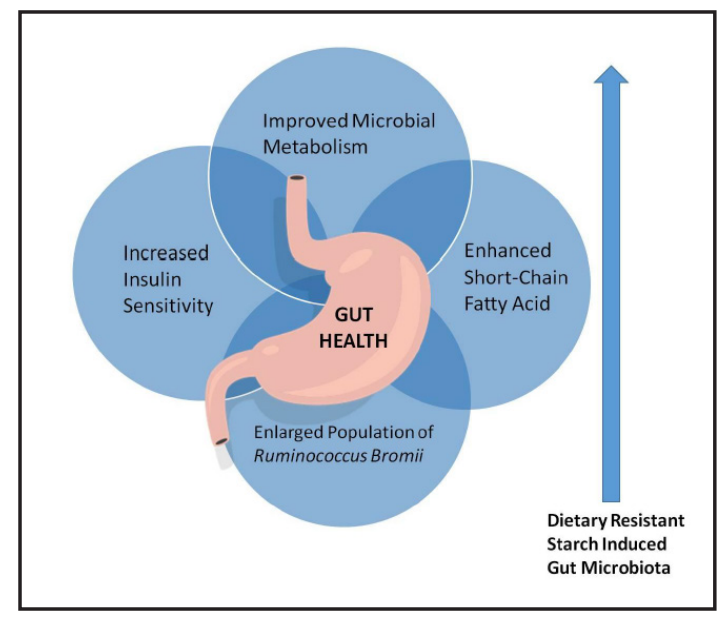

\section{Cell signaling pathways related to physiological effect of resistant starch}

A fairly amount of studies has been focused on the cell signaling pathways related to the physiological effect of resistant starch. Using the chronic kidney disease model of rats, Vaziri et al. reported that high resistant starch diet significantly attenuated disease-induced interstitial fibrosis, inflammation, tubular damage, activation of NFkB, upregulation of pro-inflammatory, pro-oxidant, and pro-fibrotic molecules; impaired Nrf2 activity, downregulation of antioxidant enzymes, and disruption of colonic epithelial tight junction [21].

Using IL-10-deficient (IL-10(-/-)) mice as a model, the effect of resistant starch on inflammation in various tissues including spleen, mesenteric lymph nodes, duodenum, jejunum, ileum, and colon was evaluated [39]. Dietary resistant starch supplementation ameliorated disease activity and decreased ileal and colonic inflammatory lesions. Furthermore, resistant starch increased the IL-10-expressing cells in spleen of wild type mice. Dietary resistant starch upregulated colonic PPAR $\gamma$ expression in wild type mice [39]. Resistant starch influences Treg cells, IFN $\gamma$, and colonic PPAR $\gamma$ expression to suppress gut inflammation.

To explore the function of resistant starch, Lacorn et al., coated butyrate, one of the most useful short chain fatty acids produced via fermentation of resistant starch by microorganisms with inulin and delivered to the distal part of the intestine of pigs. Comparing to controls, the authors observed the following phenomena: (1) coating was digested microbially in the ileum; (2) mRNA of monocarboxylate transporter-1showed a higher expression in the ileum; (3) apoptosis was reduced in the ileum but mitosis was not changed; and (4) length of villi increased by approximately $25 \%$ in the ileum [40].

Inflammatory bowel diseases including ulcerative colitis and Crohn's disease are chronic relapsing inflammatory disorders of the gastrointestinal tract. The interaction between a disturbed microbial composition, the intestinal mucosal barrier and the mucosal immune system plays an important role in inflammatory bowel diseases and its chronicity. Hartog et al. reported that oral intake with the major component of resistant starch counteracts inflammatory bowel diseases-like intestinal inflammation and weight loss in dextran sodium sulphate treated mice [41]. This reduction in inflammation might be brought about by the resistant starch-induced decrease in inflammatory cytokines, increase in IL-10 and the relative increase in Treg cells in the mesenteric lymph nodes.

Sagar et al used a combinational approach of Bifido bacterium breve with non-digestible oligosaccharides, this is, resistant starch, to explore their effects on airway inflammation and remodeling, based on a murine ovalbumin-induced chronic asthma model [42].The combination of B. breve with non-digestible oligosaccharides suppressed pulmonary airway inflammation; reduced $\mathrm{T}$ cell activation and mast cell degranulation; modulated expression of pattern recognition receptors, cytokines and transcription factors; and reduced airway remodeling. The treatment induced regulatory $\mathrm{T}$ cell responsesby increasing 


\section{Cellular Physiology Cell Physiol Biochem 2017;42:306-318 \\ \begin{tabular}{ll|l} 
and Biochemistry Published onlıne: May 22, 2017 & $\begin{array}{l}\text { (c) } 2017 \text { The Author(s). Published by S. Karger AG, Basel } \\
\text { www.karger.com/cpb }\end{array}$ \\
\hline
\end{tabular} \\ Yang et al.: Resistant Starch Regulates Gut Microbiota to Control Nutritional Events}

Il10 and Foxp3 transcription in lung tissue, and augmented Foxp3 protein expression in blood CD4+CD25+Foxp3+ T cells. This specific combination of beneficial bacteria with non-digestible oligosaccharides has strong anti-inflammatory properties, possibly via the induction of a regulatory $\mathrm{T}$ cell response, resulting in reduced airway remodeling and, therefore, may be beneficial in the treatment of chronic inflammation in allergic asthma.

Hira et al. examined the effect of continuous feeding of resistant maltodextrin to rats on GLP-1 levels and glycaemic control fasting GLP-1 levels were significantly higher in the $5 \%$ resistant maltodextrin group than in the control group. The IPGTT results showed that the glycaemic response was lower in the $5 \%$ resistant maltodextrin group than in the control group. Lower caecal $\mathrm{pH}$, higher caecal tissue and content weights were observed in the resistant maltodextrin and fructo-oligosaccharides groups. Proglucagon mRNA levels were increased in the caecum and colon of both resistant maltodextrin and fructo-oligosaccharides groups, whereas caecal GLP-1 content was increased in the $5 \%$ resistant maltodextrin group. In addition, to the aforementioned resistant maltodextrin exposure induced GLP-1 secretion in an enteroendocrine L-cell model, and single oral administration of RMD increased plasma GLP-1 levels in conscious rats. All data demonstrates that continuous ingestion of resistant maltodextrin increased GLP-1 secretion and production in normal rats, which could be stimulated by its direct and indirect (enhanced gut fermentation) effects on GLP-1-producing cells, and contributes to improving glucose tolerance [43].

Inulin-type fructans can modulate calcium absorption because they are resistant to hydrolysis by mammalian enzymes and are fermented in the large intestine to produce shortchain fatty acids, which in turn reduce luminal $\mathrm{pH}$ and modify calcium speciation, and hence solubility, or exert a direct effect on the mucosal transport pathway. Coxam et al. reported that quite a few intervention studies showed an improvement of calcium absorption in adolescents or young adults by inulin-type fructans [44].

More evidence has showed that resistant starch stimulates the secretion of glucagonlike peptide-1and peptide YYin rodents. GLP-1 and PYY are gut-secreted hormones with anti-obesity effect. Blocking the signals of increased GLP-1 and PYY may also block the effect of dietary RS on body fat. Zhou et al. utilized mouse animals of wild type C57BL/6J and knock-out GLP-1 receptor null (GLP-1R KO) fed with control or 30\% RS diet under the presence or absence of PYY receptor antagonist (BIIE0246). The results demonstrated that dietary resistant starch significantly decreased body fat accumulation only in wild-type mice with injection of saline, but not in GLP-1R KO mice. PYY receptor antagonist diminished resistant starch action on body fat in wild-type mice, but did not interfere with GLP-1R KO mice response to resistant starch. Regardless of genotype and injection received, all resistant starch-fed mice had increased cumulative food intake, cecal fermentation, and mRNA expression of proglucagon and PYY. These data convince that increased GLP-1 and PYY is important in resistant starch effects on body fat accumulation [45].

To investigate the influence of resistant starch on inflammation-associated nutritional functions, Valcheva et al., developed interleukin 10-deficient (IL-10(-/-)) mice for establishing a colitis animal model that is influenced by the gut microbial composition. The results showed that there were no significant differences in mouse growth, intestinal weight, length, or gut permeability with feeding resistant starch. Mice fed resistant starch secreted $47-88 \%$ less colonic IL-1 $\beta$, tumor necrosis factor $\alpha$, and IL-23 and IL-12 heterodimer p70, IL-6, and chemokine ligand 1 (CXCL1) than did the control group, indicating the robust effect of the resistant starch on inflammation-related cytokines and chemokines. Functionally, the resistant starch diet group had a lower enterocyte injury score than did control mice, and this was associated with increased abundance of butyrate producers, including Incertae sedis XIV, Lachnospiraceae, and Ruminococcaceae, demonstrating the microbiota alterations induced by resistant starch [46].

As previously mentioned, resistant starch treatment is associated with increased gut hormones peptide YY (PYY) and glucagon-like peptide 1 (GLP-1), Vidrine et al. was trying to investigate whether sodium butyrate exerts any effect on alterations of these hormones. They found that both resistant starch and sodium butyrate reduced more abdominal fat and 


\section{Cellular Physiology Cell Physiol Biochem 2017;42:306-318 \begin{tabular}{ll|l} 
DOI: 10.1159/000477386 & O 2017 The Author(s). Published by S. Karger AG, Basel \\
www.karger.com/cpb
\end{tabular} \\ Yang et al.: Resistant Starch Regulates Gut Microbiota to Control Nutritional Events}

the combination reduced abdominal fat compared to sodium butyrate and resistant starch. Resistant starch increased the levels of serum PYY and GLP-1. Production of cecal butyrate was increased from the fermentation of resistant starch rather than from dietary sodium butyrate. These results imply that sodium butyrate after its absorption into the blood appears to not affect production of PYY and GLP-1, while butyrate from fermentation in the cecum promotes increased PYY and GLP-1 [47]. These observations are meaningful since localization of butyrate in gut rather than in blood, is very important in initiating the cell signaling pathway by secreting the PYY and GLP-1.

Based on the results of 19 young adults supplied with either boiled barley kernels (resistant starch) or white wheat bread, analysis of glucose, plasma insulin, adiponectin, ghrelin, glucose-dependent insulinotropic polypeptide and glucagon-like peptide-1, serum free fatty acids and interleukin (IL)-6 [48]. Resistant starch diet increased plasma GLP-1, decreased fasting serum free fatty acids and decreased fasting serum IL- 6 levels. This diet decreased voluntary energy intake, decreased incremental blood glucose area, promoted higher breath $\mathrm{H} 2$, maintained adiponectin concentrations and reduced perceived hunger. The results clearly indicated that resistant starch facilitates glucose regulation, increases the release of GLP-1, reduces subsequent energy intake while at the same time decreasing hunger over 2 subsequent meals, and reduce fasting FFA in the subsequent morning.

Zhou et al. found that resistant starch stimulates GLP-1 and PYY secretion in a substantial day-long manner, independent of meal effect or changes in dietary glycemia [49]. Fermentation and the liberation of short-chain fatty acids in the lower gut are associated with increased proglucagon and PYY gene expression. Glucose tolerance, an indicator of increased active forms of GLP-1 and PYY, is improved in resistant starch-fed diabetic mice. Fermentation of resistant starch is most likely the primary mechanism for increased endogenous secretions of total GLP-1 and PYY in rodents.

Using 18-20 months old healthy mice, Zhou et al. examined the effects of dietary resistant starch on tolerance, colonic fermentation, and cytokine expression. PYY mRNA expression as fermentation indicators are enhanced in a resistant starch dose-dependent manner. No difference of serum concentrations of soluble cytokine receptors (sTNF-Rb, sIL4R, sIL-2R $\alpha$, sVEGFR1, and sRAGE) and TNF $\alpha$ expression (gene and protein) among three groups in visceral were observed. This study delivers an important message that dietary resistant starch is well tolerated, fermented in the colon, and stimulates colonic expression of proglucagon and PYY mRNA, and adiponectin protein in visceral fat for aged rodents [50].

Supplying resistant starch is able to increase production of short-chain fatty acid, representing an important approach to treat acute diarrhea in children less than five years of age [51]. Principally, nonabsorbed carbohydrates are not absorbed in the colon and result in an osmotically mediated diarrhea. In contrast, short-chain fatty acids are absorbed by colonic epithelial cells and stimulate Na-dependent fluid absorption via a cyclic AMP-independent process involving apical membrane $\mathrm{Na}-\mathrm{H}$, short-chain fatty acid-HCO(3), and $\mathrm{Cl}$-short-chain fatty acid exchanges. Thus, diets with resistant starch exert therapeutic and protective roles in treating the diarrhea. Brief idenfied cell signaling pathways are summarized in Fig. 3.

Fig. 3. Consumption of resistant starch increases the expression of PYY and GLP-1 and reduces the levels of IL-6 and FFA via either IL-10 inflammatory or GLP-1R secreted hormone.

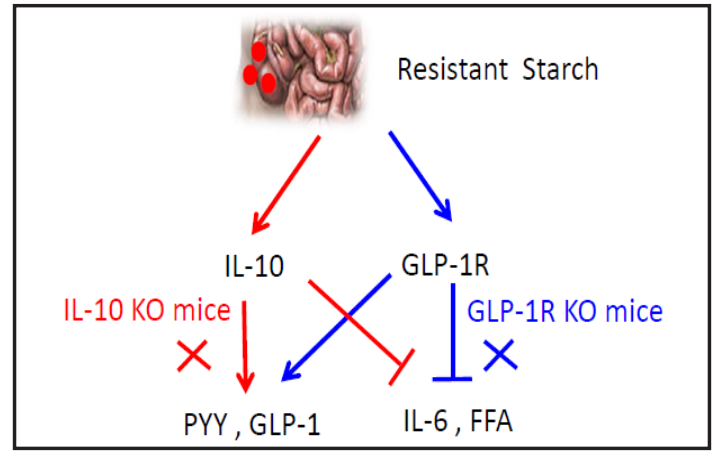




\section{Cellular Physiology Cell Physiol Biochem 2017;42:306-318 and Biochemistry Published onlIne: May 22, $2017 \quad \begin{aligned} & \text { DOI: 10.1159/000477386 } 2017 \text { The Author(s). Published by S. Karger AG, Basel } \\ & \text { www.karger.com/cpb }\end{aligned}$ Yang et al.: Resistant Starch Regulates Gut Microbiota to Control Nutritional Events}

\section{Potential side effect}

Since the potential application of RS has been raised, possible side effects for this type of food have to be investigated. An early report showed that RS had no side effect on health [52]. Recent results suggest that resistant starch acts through desmutagenesis and bio-antimutagenesis, as well as by reducing aberrant crypt foci, thereby improving disease prognosis [53]. In a Msh2-deficient mouse model, RS can reduce red meat-induced promutagenic lesions and chemically induced colon tumours [54].

There was a well designed study which explored the potential side effect of RS using the transgenic rice line enriched with amylose and resistant starch developed by antisense RNA inhibition of starch-branching enzymes. The authors determined the effect of transgenic rice on rats by feeding a diet containing $70 \%$ transgenic rice flour. Clinical performance, reproductive capacity and pathological responses including body weight, food consumption, reproductive data, hematological parameters, serum chemistry components, organ relative weights and histopathology of these rats were examined. No adverse effect was observed in most of the clinical pathological parameters among the rats fed the transgenic rice diet, including hematological parameters, serum components and urinalysis. No difference of reproductive capacity of F3 males and females rats and serum hormone was observed either. . No evidence of altered incidence or altered severity of pathological changes or lesions was seen in any organs and tissues of male and female rats subjected to the feeding of transgenic rice diet as well [55].

Consumption of resistant starch can attenuate disruption of vitamin D homeostasis in type I diabetes through the rescue of megalin-mediated endocytosis in kidney in the absence of visible side effects [56].

\section{Conclusion}

Resistant starch (RS) is a newly recognized healthy food for both human and animals. Due to its content of high ratio amylose and low ratio of amylopectin, RS improves the population of gut microbiota and triggers cell signaling pathway associated with antiinflammation, anti-diabetics and anti-obesity. Probiotics is a new concept for preventing metabolic diseases in human beings and producing high quality of meat in animal nutrition. However, these theoretical studies are in the very early phase far behind application. Further study is warranted for a better understanding of using RS as a probiotics. Taking home message is listed in Table 1.

\section{Abbreviations}

AMP (Adenosine monophosphate); CKD (Chronic Kidney Disease); CXCL 1 (chemokine ligand 1); ESI-MS (Electrospray Ionization Mass Spectrometry); FFA (Free Fatty Acids); GCG (Glucagon); GLP-1 (Glucagon-like peptide 1); hs-CRP (High Sensitive C-Reactive Protein); IFN $\gamma$ (Interferon gamma); IGF-1 (Insulin-like Growth Factor); IL (Interleukin); IPGTT (Intraperitoneal Glucose Tolerance Test); MDA (Malondialdehyde); MMP-2 (Matrix Metallopeptidase 2); PPAR $\gamma$ (Peroxisome Proliferator-Activa-

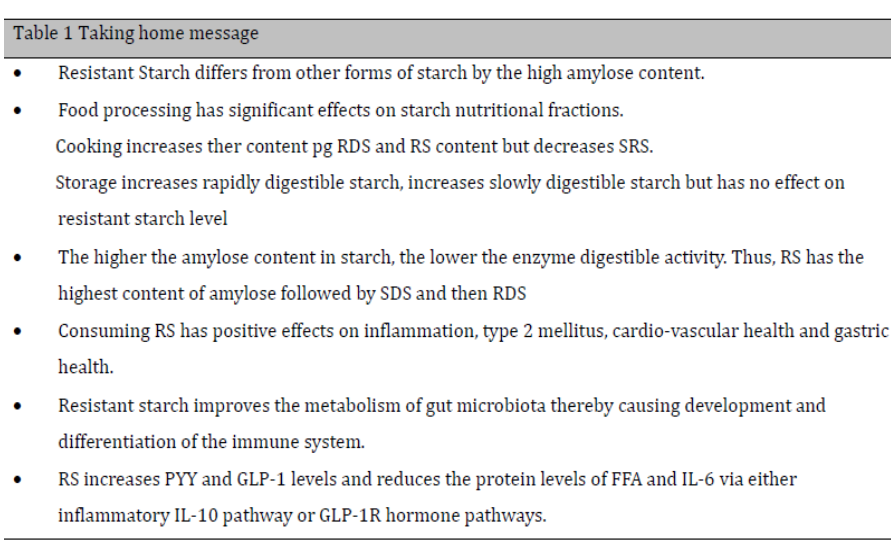




\section{Cellular Physiology Cell Physiol Biochem 2017;42:306-318 \begin{tabular}{l|l|l} 
and Biochemistry.1159/000477386 & $\begin{array}{l}\text { (c) 2017 The Author(s). Published by S. Karger AG, Basel } \\
\text { www.karger.com/cpb }\end{array}$
\end{tabular}}

Yang et al.: Resistant Starch Regulates Gut Microbiota to Control Nutritional Events

ted Receptors Gamma); PYY (Peptide YY); RDS (Rapid Digestive Starch); RS (Rapid Starch); SCFA (Short Chain Fatty Acids); SDS (Slowly Digestion Starch); TAC (Total Antioxidant Capacity); TIMP-2 (Tissue Inhibitor of Metalloproteinases-2).

\section{Acknowledgement}

This project was supported by The National Key Research and Development Program of China (2016YFD0501201) to Y. Y., H. Y. and X. Y. , the Key Programs of Frontier Scientific Research of the Chinese Academy of Sciences (QYZDY-SSW- SMC008) to Y. Y. and Xiaoxiang Endowed University Professor Fund of Hunan Normal University (No. 840140-008) to X.Y.

\section{Disclosure Statement}

No conflict of interets.

\section{References}

$\rightarrow$ Englyst H, Wiggins HS, Cummings JH: Determination of the non-starch polysaccharides in plant foods by gas-liquid chromatography of constituent sugars as alditol acetates. Analyst 1982;107:307-318.

-2 Trinh KS:Recrystallization of starches by hydrothermal treatment: digestibility, structural, and physicochemical properties. J Food Sci Technol 2015;52:7640-7654.

- Haralampu SG: Resistant starch - a review of the physical properties and biological impact of RS 3. Carbohydr Polym 2000;41:285-292.

4 Yin YL, Deng ZY, Huang HL, Zhong HY, Hou ZP, Gong J, Liu Q: Nutritional and health functions of carbohydrate for pigs. J Anim Feed Sci 2004;13:523-538.

-5 Nunes FM, Lopes ES, Moreira AS, Simões J, Coimbra MA, Domingues RM: Formation of type 4 resistant starch and maltodextrins from amylose and amylopectin upon dry heating: A model study. Carbohydr Polym 2016;141:253-262.

6 Kong X, Chen Y, Zhu P, Sui Z, Corke H, Bao J: Relationships among genetic, structural, and functional properties of rice starch. J Agric Food Chem 2015;63:6241-6248.

7 Van Hung P, Vien NL, Phi NT: Resistant starch improvement of rice starches under a combination of acid and heat-moisture treatments. Food Chem 2016;191:67-73.

8 Rashmi S, Urooj A: Effect of processing on nutritionally important starch fractions in rice varieties. Int J Food Sci Nutrv 2003;54:27-36.

-9 Panlasigui LN, Thompson LU, Juliano BO, Perez CM, Yiu SH, Greenberg GR: Rice varieties with similar amylose content differ in starch digestibility and glycemic response in humans. Am J Clin Nutr 1991;54:871-877.

10 Man J, Lin L, Wang Z, Wang Y, Liu Q, Wei C: Different structures of heterogeneous starch granules from highamylose rice. J Agric Food Chem 2014;62:11254-11263.

11 Bindels LB, Walter J, Ramer-Tait AE: Resistant starches for the management of metabolic diseases. Curr Opin Clin Nutr 2015;18:559-565.

12 Heo JM, Agyekum AK, Yin YL, Rideout TC, Nyachoti CM: Feeding a diet containing resistant potato starch influences gastrointestinal tract traits and growth performance of weaned pigs. J Anim Sci 2014;92:39063913.

13 Karimi P, Farhangi MA, Sarmadi B, Gargari BP, Zare Javid A, Pouraghaei M, Dehghan P: The Therapeutic Potential of Resistant Starch in Modulation of Insulin Resistance, Endotoxemia, Oxidative Stress and Antioxidant Biomarkers in Women with Type 2 Diabetes: A Randomized Controlled Clinical Trial. Ann Nutr Metab 2016;68:85-93.

14 Sun Y, Yu K, Zhou L, Fang L, Su Y, Zhu W: Metabolomic and transcriptomic responses induced in the livers of pigs by the long-term intake of resistant starch. J Anim Sci 2016;94:1083-1094. 


\section{Cellular Physiology Cell Physiol Biochem 2017;42:306-318 \begin{tabular}{l|l} 
DOI: 10.1159/000477386 & O 2017 The Author(s). Published by S. Karger AG, Basel \\
www.karger.com/cpb
\end{tabular} \\ Yang et al.: Resistant Starch Regulates Gut Microbiota to Control Nutritional Events}

15 Messina V: Nutritional and health benefits of dried beans. Am J Clin Nutr 2014;100:437S-442S.

16 Shoaib M, Shehzad A, Omar M, Rakha A, Raza H, Sharif HR, Shakeel A, Ansari A, Niazi S: Inulin: Properties, health benefits and food applications. Carbohydr Polym 2016;147:444-454.

17 Bernstein AM, Titgemeier B, Kirkpatrick K, Golubic M, Roizen MF: Major cereal grain fibers and psyllium in relation to cardiovascular health. Nutrients 2013;5:1471-1487.

18 Le Leu RK, Young GP, Hu Y, Winter J, Conlon MA: Dietary red meat aggravates dextran sulfate sodiuminduced colitis in mice whereas resistant starch attenuates inflammation. Dig Dis Sci 2013;58:3475-3482.

19 Kwak JH, Paik JK, Kim HI, Kim OY, Shin DY, Kim HJ, Lee JH, Lee JH: Dietary treatment with rice containing resistant starch improves markers of endothelial function with reduction of postprandial blood glucose and oxidative stress in patients with prediabetes or newly diagnosed type 2 diabetes. Atherosclerosis 2012;224:457-464.

20 Johnston KL, Thomas EL, Bell JD, Frost GS, Robertson MD: Resistant starch improves insulin sensitivity in metabolic syndrome. Diabet Med 2010;27:391-397.

21 Vaziri ND, Liu SM, Lau WL, Khazaeli M, Nazertehrani S, Farzaneh SH, Kieffer DA, Adams SH, Martin RJ: High amylose resistant starch diet ameliorates oxidative stress, inflammation, and progression of chronic kidney disease. PloS one 2014;9:e114881.

22 Upadhyaya B, McCormack L, Fardin-Kia AR, Juenemann R, Nichenametla S, Clapper J, Specker B, Dey M: Impact of dietary resistant starch type 4 on human gut microbiota and immunometabolic functions. Sci Rep 2016;6:28797.

23 Sun Y, Su Y, Zhu W: Microbiome-metabolome responses in the cecum and colon of pig to a high resistant starch diet. Front Microbiol 2016;7:779.

-24 Deng J, Wu X, Bin S, Li TJ, Huang R, Liu Z, Liu Y, Ruan Z, Deng Z, Hou Y, Yin YL: Dietary amylose and amylopectin ratio and resistant starch content affects plasma glucose, lactic acid, hormone levels and protein synthesis in splanchnic tissues. J Anim Physiol Anim Nutr 2010 ;94:220-226.

-25 Tang ZR, Yin YL, Nyachoti CM, Huang RL, Li TJ, Yang C, Yang XJ, Gong J, Peng J, Qi DS, Xing JJ: Effect of dietary supplementation of chitosan and galacto-mannan-oligosaccharide on serum parameters and the insulinlike growth factor-I mRNA expression in early-weaned piglets. Domest Anim Endocrinol 2005;28:430-441.

26 Huang B, Xiao D, Tan B, Xiao H, Wang J, Yin J, Duan J, Huang R, Yang C, Yin Y: Chitosan Oligosaccharide Reduces Intestinal Inflammation That Involves Calcium-Sensing Receptor (CaSR) Activation in Lipopolysaccharide (LPS)-Challenged Piglets. J Agric Food Chem 2015;64:245-252.

27 Lyte M, Chapel A, Lyte JM, Ai Y, Proctor A, Jane JL, Phillips GJ: Resistant Starch Alters the Microbiota-Gut Brain Axis: Implications for Dietary Modulation of Behavior. PloS one 2016;11:e0146406.

28 Toden S, Belobrajdic DP, Bird AR, Topping DL, Conlon MA: Effects of dietary beef and chicken with and without high amylose maize starch on blood malondialdehyde, interleukins, IGF-I, insulin, leptin, MMP-2, and TIMP-2 concentrations in rats. Nutr Cancer 2010;62:454-465.

29 Roeselers G, Ponomarenko M, Lukovac S, Wortelboer HM: Ex vivo systems to study host-microbiota interactions in the gastrointestinal tract. Best Pract Res Clin Gastroenterol 2013;27:101-113.

30 Flint HJ, Scott KP, Duncan SH, Louis P, Forano E: Microbial degradation of complex carbohydrates in the gut. Gut microbes 2012;3:289-306.

- 31 Venkataraman A, Sieber JR, Schmidt AW, Waldron C, Theis KR, Schmidt TM: Variable responses of human microbiomes to dietary supplementation with resistant starch. Microbiome 2016;4:33.

-32 Ze X, David YB, Laverde-Gomez JA, Dassa B, Sheridan PO, Duncan SH, Louis P, Henrissat B, Juge N, Koropatkin NM, Bayer EA: Unique organization of extracellular amylases into amylosomes in the resistant starch-utilizing human colonic Firmicutes bacterium Ruminococcus bromii. MBio 2015;6:e01058-1015.

33 Edwards CH, Grundy MM, Grassby T, Vasilopoulou D, Frost GS, Butterworth PJ, Berry SE, Sanderson J, Ellis PR: Manipulation of starch bioaccessibility in wheat endosperm to regulate starch digestion, postprandial glycemia, insulinemia, and gut hormone responses: a randomized controlled trial in healthy ileostomy participants. Am J Clin Nutr 2015;102:791-800.

-34 Bindels LB, Neyrinck AM, Salazar N, Taminiau B, Druart C, Muccioli GG, François E, Blecker C, Richel A, Daube G, Mahillon J: Non digestible oligosaccharides modulate the gut microbiota to control the development of leukemia and associated cachexia in mice. PloS one 2015;10:e0131009.

- 35 Lange K, Hugenholtz F, Jonathan MC, Schols HA, Kleerebezem M, Smidt H, Müller M, Hooiveld GJ: Comparison of the effects of five dietary fibers on mucosal transcriptional profiles, and luminal microbiota composition and SCFA concentrations in murine colon. Mol Nutr Food Res 2015;59:1590-1602. 


\section{Cellular Physiology Cell Physiol Biochem 2017;42:306-318 \begin{tabular}{l|l|l} 
and Biochemistry 10.1159/000477386 & $\begin{array}{l}\text { D } 2017 \text { The Author(s). Published by S. Karger AG, Basel } \\
\text { www.karger.com/cpb }\end{array}$
\end{tabular} \\ Yang et al.: Resistant Starch Regulates Gut Microbiota to Control Nutritional Events}

-36 Nilsson AC, Johansson-Boll EV, Björck IM: Increased gut hormones and insulin sensitivity index following a 3-d intervention with a barley kernel-based product: a randomised cross-over study in healthy middleaged subjects. Br J Nutr 2015;114:899-907.

-37 Haenen D, Zhang J, da Silva CS, Bosch G, van der Meer IM, van Arkel J, van den Borne JJ, Gutiérrez OP, Smidt H, Kemp B, Müller M: A diet high in resistant starch modulates microbiota composition, SCFA concentrations, and gene expression in pig intestine. J Nutr 2013;143:274-283.

38 Flint HJ, Scott KP, Duncan SH, Louis P, Forano E: Microbial degradation of complex carbohydrates in the gut. Gut microbes 2012;3:289-306.

-39 Bassaganya-Riera J, DiGuardo M, Viladomiu M, de Horna A, Sanchez S, Einerhand AW, Sanders L, Hontecillas R: Soluble fibers and resistant starch ameliorate disease activity in interleukin-10-deficient mice with inflammatory bowel disease. J Nutr 2011;141:1318-1325.

-40 Lacorn M, Goerke M, Claus R: Inulin-coated butyrate increases ileal MCT1 expression and affects mucosal morphology in the porcine ileum by reduced apoptosis. J Anim Physiol Anim Nutr 2010;94:670-676.

- 41 Hartog A, Belle FN, Bastiaans J, de Graaff P, Garssen J, Harthoorn LF, Vos AP: A potential role for regulatory T-cells in the amelioration of DSS induced colitis by dietary non-digestible polysaccharides. J Nutr Biochem 2015;26:227-233.

42 Sagar S, Vos AP, Morgan ME, Garssen J, Georgiou NA, Boon L, Kraneveld AD, Folkerts G: The combination of Bifidobacterium breve with non-digestible oligosaccharides suppresses airway inflammation in a murine model for chronic asthma. Biochim Biophys Acta (BBA)-Molecular Basis of Disease 2014;1842:573-583.

-43 Hira T, Ikee A, Kishimoto Y, Kanahori S, Hara H: Resistant maltodextrin promotes fasting glucagon-like peptide-1 secretion and production together with glucose tolerance in rats. Br J Nutr 2015;114:34-42.

44 Coxam V: Current data with inulin-type fructans and calcium, targeting bone health in adults. J Nutr 2007; 137:2527S-2533S.

45 Zhou J, Martin RJ, Raggio AM, Shen L, McCutcheon K, Keenan MJ: The importance of GLP-1 and PYY in resistant starch's effect on body fat in mice. Mol Nutr Food Res 2015;59:1000-1003.

46 Valcheva R, Hotte N, Gillevet P, Sikaroodi M, Thiessen A, Madsen KL: Soluble Dextrin Fibers Alter the Intestinal Microbiota and Reduce Proinflammatory Cytokine Secretion in Male IL-10-Deficient Mice. J Nutr 2015;145:2060-2066.

47 Vidrine K, Ye J, Martin RJ, McCutcheon KL, Raggio AM, Pelkman C, Durham HA, Zhou J, Senevirathne RN, Williams C, Greenway F: Resistant starch from high amylose maize (HAM-RS2) and Dietary butyrate reduce abdominal fat by a different apparent mechanism. Obesity 2014;22:344-348.

48 Johansson EV, Nilsson AC, Östman EM, Björck IM: Effects of indigestible carbohydrates in barley on glucose metabolism, appetite and voluntary food intake over $16 \mathrm{~h}$ in healthy adults. J Nutr 2013;12:1.

-49 Zhou J, Martin RJ, Tulley RT, Raggio AM, McCutcheon KL, Shen L, Danna SC, Tripathy S, Hegsted M, Keenan MJ: Dietary resistant starch upregulates total GLP-1 and PYY in a sustained day-long manner through fermentation in rodents. Am J Physiol Endocrinol Metab 2008;295:E1160-1166.

-50 Zhou J, Keenan MJ, Keller J, Fernandez-Kim SO, Pistell PJ, Tulley RT, Raggio AM, Shen L, Zhang H, Martin RJ, Blackman MR: Tolerance, fermentation, and cytokine expression in healthy aged male C57BL/6J mice fed resistant starch. Mol Nutr Food Res 2012;56:515-518.

-51 Binder HJ: Role of colonic short-chain fatty acid transport in diarrhea. Annu Rev Physiol 2010;72:297-313. Goldring JM: Resistant starch: safe intakes and legal status. J AOAC Int 2004;87:733-739.

-53 Navarro SD, Mauro MO, Pesarini JR, Ogo FM, Oliveira RJ: Resistant starch: a functional food that prevents DNA damage and chemical carcinogenesis. Genet Mol Biol 2015;14:1679-1691.

-54 Winter JM, Hu Y, Young GP, Kohonen-Corish MR, Le Leu RK: Role of red meat and resistant starch in promutagenic adduct formation, MGMT repair, thymic lymphoma and intestinal tumourigenesis in Msh2deficient mice. J Nutrigenet Nutrigenomics 2015;7:299-313.

-55 Zhou XH, Dong Y, Zhao YS, Xiao X, Wang Y, He YQ, Liu QQ: A three generation reproduction study with Sprague-Dawley rats consuming high-amylose transgenic rice. Food Chem Toxicol 2014;74:20-27.

-56 Smazal AL, Borcherding NC, Anderegg AS, Schalinske KL, Whitley EM, Rowling MJ: Dietary resistant starch prevents urinary excretion of 25-hydroxycholecalciferol and vitamin D-binding protein in type 1 diabetic rats. J Nutr 2013;143:1123-1128. 